\title{
Trabecular Meshwork
}

National Cancer Institute

\section{Source}

National Cancer Institute. Trabecular Meshwork. NCI Thesaurus. Code C12803.

Mesh-like tissue responsible for draining the aqueous humor from the eye that is located at the iridocorneal ang le of the eye, between the anterior chamber and the venous sinus of the sclera. 